\title{
Near-Field Characteristics of a TEM Horn Used for Radi- ated Immunity Tests
}

\author{
Katsushige Harima $^{1 \mathrm{a})}$, Takayuki Kubo ${ }^{2}$, Kaoru Gotoh ${ }^{1}$, \\ and Takeshi Ishida ${ }^{2}$ \\ ${ }^{1}$ National Institute of Information and Communications Technology \\ 4-2-1, Nukui-kitamachi, Koganei, Tokyo 184-8795, Japan \\ 2 Noise Laboratory Co., Ltd. \\ 1-4-4, Chiyoda, Chuo-ku, Sagamihara, Kanagawa 252-0237, Japan \\ a) harima@nict.go.jp
}

\begin{abstract}
We have fabricated a transverse electromagnetic (TEM) horn for radiated immunity tests in close proximity as specified in standard IEC 61000-4-39 and compared its near-field characteristics with those of other typical test antennas such as broadband dipole and double-ridged guide (DRG) horn antennas. Experimental results show that the TEM horn generates a homogeneous field and maintains the field strength without a rapid change near the antenna.
\end{abstract}

Keywords: broadband dipole, immunity, radiated immunity test in close proximity, phase center, TEM horn, wave impedance

Classification: Electromagnetic compatibility (EMC)

\section{References}

[1] IEC 61000-4-39, Electromagnetic compatibility (EMC) - Part 4-39: Testing and measurement techniques - Radiated fields in close proximity Immunity test, 2017.

[2] ISO 11452-9, Road vehicles - Component test methods for electrical disturbances from narrowband radiated electromagnetic energy - Part 9: Portable transmitters, 2012.

[3] K. Harima, T. Kubo, T. Ishida, "Evaluation of a TEM horn antenna for radiated immunity tests in close proximity," IEICE Communications Express, vol. 9, no. 2, pp. 60-65, Feb. 2020. DOI:10.1587/comex. 2019XBL0137

[4] M. Manteghi and Y. Rahmat-Samii, "A novel UWB feeding mechanism for the TEM horn antenna, reflector IRA, and the Vivaldi antenna," IEEE Antennas Propag. Mag., vol. 46, no. 5, pp. 81-87, Oct. 2004. DOI:10.1109/ MAP.2004.1388832

[5] K. Harima, "Numerical simulation of far-field gain determination at reduced distances using phase center," IEICE Trans. Commun., vol. E97-B, no. 10, pp. 2001-2010, Oct. 2014. DOI:10.1587/transcom.E97.B.2001

[6] A. R. Panicali and M. M. Nakamura, "On the amplitude center of radiating apertures," IEEE Trans. Antennas Propag., vol. AP-33, no. 3, pp. 330-335, Mar. 1985. 10.1109/TAP.1985.1143572

[7] CST Studio Suite, Dassault systems. 


\section{Introduction}

Radiated immunity tests related to electromagnetic fields of electronic equipment or systems have been performed under far-field conditions. Recently, with the widespread use of portable wireless devices such as mobile phones, immunity requirements for electronic equipment with the aim of ensuring protection against portable transmitters used in close proximity have been specified in international standards $[1,2]$ and manufacturer standards. In near-field radiated immunity tests, TEM horns and dipole-like antennas such as sleeve antennas, monopole antennas, and broadband dipoles are used in accordance with test standards. The field uniformity and the propagation characteristics in proximity to the antenna affect the field strength reaching the active electronic components inside the electronic equipment depending on used antenna, even when the field strength is specified in the illuminated surface of the electronic equipment.

In this study, we fabricated a TEM horn that complies with IEC standards for proximity immunity tests [1]. The field characteristics of the TEM horn near the antenna were compared with those of the flat broadband dipole used in near-field immunity tests in automotive industry [2], the half-wave dipole used as a basic antenna, and the DRG horn widely used in EMC measurements. In addition, the far-field approximation from the characteristics of propagation over a distance and the wave impedance were evaluated considering the phase center.

\section{TEM horn}

The radiated immunity test in close proximity is carried out in a frequency range of $380 \mathrm{MHz}$ to $6 \mathrm{GHz}$, which is used in portable transmitters such as mobile phones. The field-generating antenna is placed in a position $100 \mathrm{~mm}$ from the front of the equipment under test (EUT) and illuminates it with a specified field strength of $10 \mathrm{~V} / \mathrm{m}$ to $300 \mathrm{~V} / \mathrm{m}$ [1].

A shortened exponentially tapered TEM horn [3] we fabricated is shown in Figs. 1(a) and (b). The plate has a tapered structure designed to maintain the characteristic impedance of the exponential taper transmission line considering the matching from the feeding section to the aperture. The antenna length was decreased by $10 \%$ to improve the radiation directivity. Although the TEM horn is a balanced antenna, the leakage current flowing outside the outer conductor of the coaxial cable is suppressed using the balanced feeding mechanism without using the balun circuit $[3,4]$. The 0.6 -mm-thick plates of the antenna were made of brass, and the spacing between them was maintained using an expanded hard foam with a relative dielectric constant of 1.07 .

\subsection{Phase center}

The phase center, which is defined as the center of curvature of the equiphase front in the far-field region, is effective for antenna measurements [5]. The mean of the phase centers of the electric $(\mathrm{E})$ and magnetic $(\mathrm{H})$ planes, $d_{p c}$, 


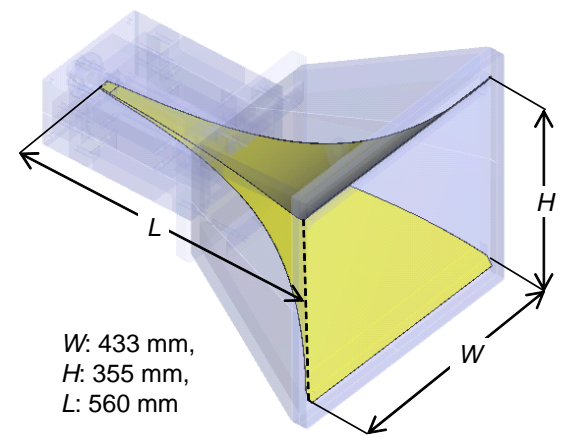

(a)

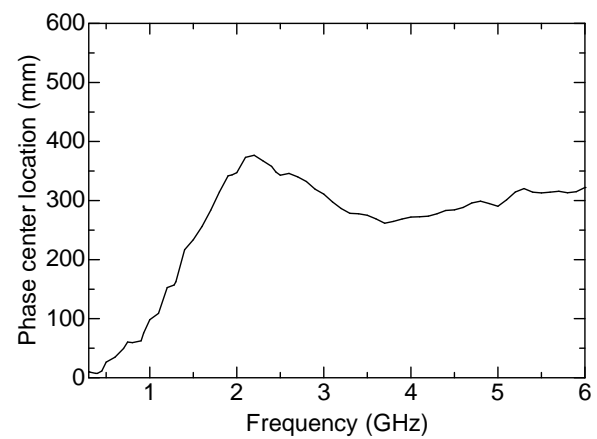

(c)

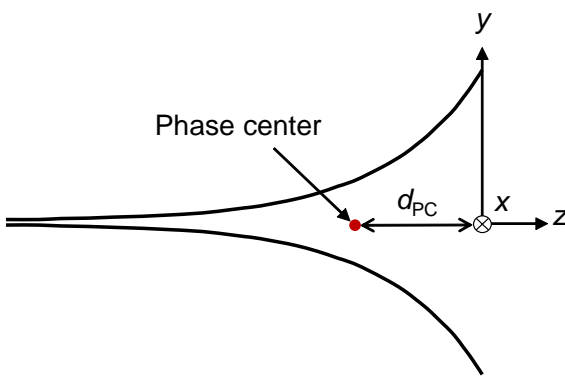

(b)

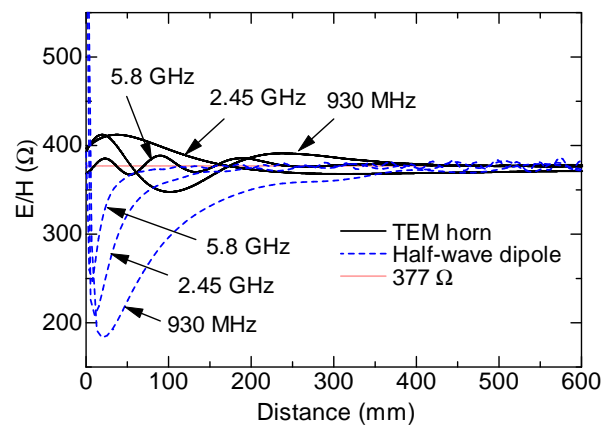

(d)

Fig. 1: (a) Internal structure and (b) cross-sectional view of TEM horn. (c) Calculated phase center location and (d) wave impedance of TEM horn.

coincides with the location of the amplitude center [6]; thus, it can be treated as an equivalent point source.

Figure 1(c) shows the calculation result of the phase center of the TEM horn. The phase center was calculated from the equiphase pattern by adjusting the origin of the near- to- far-field transformation using a CST Studio Suite solver [7] based on the finite integration technique (FIT). The phase center depends on frequency; as frequency increases, the phase center moves from the aperture to the middle of the antenna.

\subsection{Wave impedance}

The wave impedance of the electromagnetic wave radiated from the antenna is equal to the free space impedance of $377 \Omega$ when it is sufficiently far from the antenna. In other words, as one of the conditions for the far-field region, the wave impedance should satisfy the free space impedance. The wave impedance on the axis of the antenna close to the antenna was calculated as the ratio of the E- to H-fields using the FIT solver. The calculation result of the wave impedance of the TEM horn is shown in Fig. 1(d) and compared with that of half-wave dipoles in the same figure. Although the wave impedance converges toward a constant value of $377 \Omega$ as the distance from the antenna increases, it in close to the antenna differs depending on the frequency and type of the antenna. The wave impedance of the TEM 


\section{Experiments and results}

The field characteristics in proximity were evaluated for the TEM horn and other typical EMC antennas, which are half-wave dipoles, the flat broadband dipole used in near-field tests for vehicles [2], and the DRG horn often used in EMC measurements. The experimental setup is shown in Fig. 2(a). A field-generating antenna and a single-axis E-field probe were separated by the distance $r$ in a full anechoic chamber. The frequency-selective E-field probe with an optical fiber link was connected to a vector network analyzer (VNA). The E-field distribution on a plane of $400 \mathrm{~mm} \times 400 \mathrm{~mm}$ was measured along the antenna axis from $50 \mathrm{~mm}$ to $400 \mathrm{~mm}(=r)$ by scanning with the probe using an XYZ positioner at typical frequencies of $930 \mathrm{MHz}, 2.45 \mathrm{GHz}$, and $5.8 \mathrm{GHz}$ used for the immunity tests. The dimensions $(W, H$, and $L)$ of the DRG horn used for comparisons were $244 \times 159 \times 279 \mathrm{~mm}$. The broadband dipole had the flat element with dimensions of $109 \times 240 \mathrm{~mm}$, and the element lengths of half-wave dipoles were $175 \mathrm{~mm}, 67 \mathrm{~mm}$, and $29 \mathrm{~mm}$.

Figures 2(b) to (d) show the measured results of the propagation characteristics along the axis of the antenna. The received field level was normalized at $100 \mathrm{~V} / \mathrm{m}$ at a distance of $100 \mathrm{~mm}$, which is the condition used in the proximity immunity tests. The measured values of the TEM horn and half-wave dipole are in good agreement with the results calculated using the FIT solver.

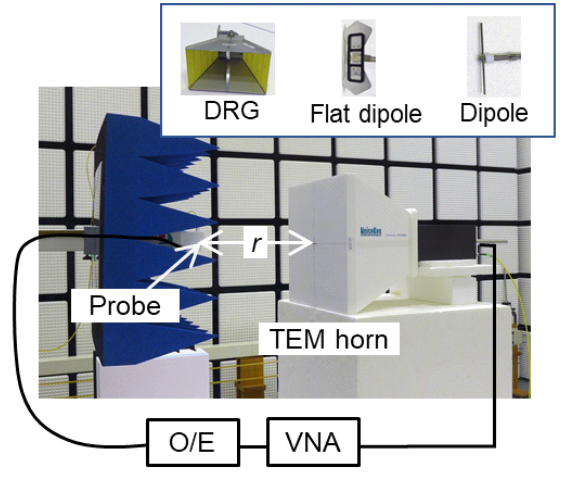

(a)

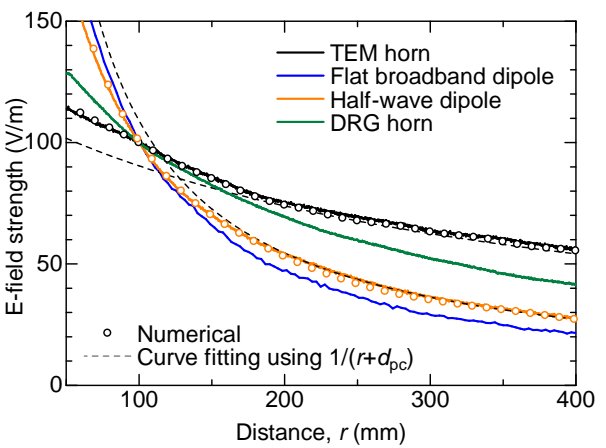

(c)

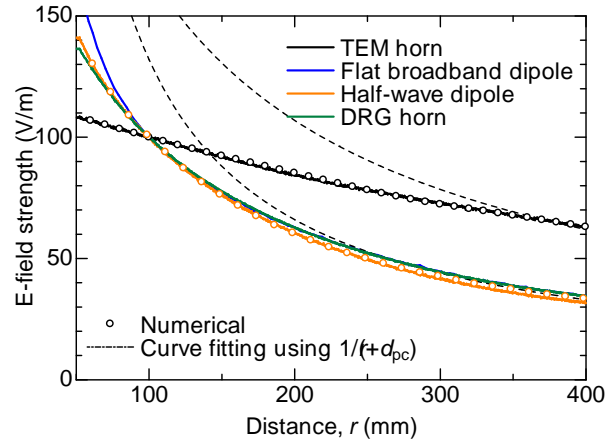

(b)

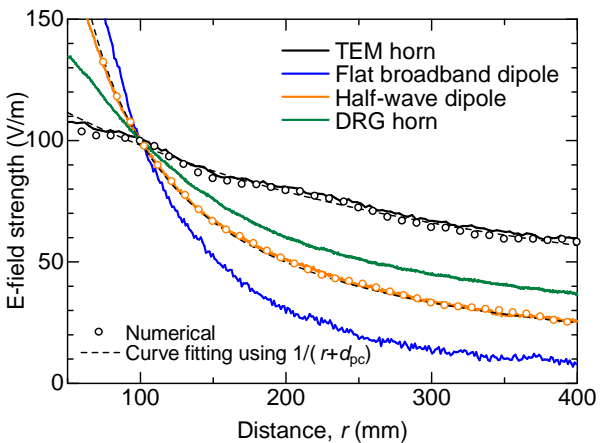

(d)

Fig. 2: (a) Experimental set-up. Field characteristics of TEM horn, halfwave dipole, flat broadband dipole, and DRG horn at (b) $930 \mathrm{MHz}$, (c) $2.45 \mathrm{GHz}$, and (d) $5.8 \mathrm{GHz}$. 
These results indicate that the field characteristics along the distance in close proximity to the antenna greatly differ among the antenna types. That is, the field strength to which the active electronic circuits are exposed inside the EUT changes depending on the antenna, even when the field strength was specified at the surface of the EUT. The TEM horn in close proximity showed field characteristics with a smaller reduction in field strength than other antennas, especially dipoles. For example, the distances where the field strength decreases by $4 \mathrm{~dB}$ from the specified position $(r=100 \mathrm{~mm})$ at $2.45 \mathrm{GHz}$ are $209 \mathrm{~mm}, 54 \mathrm{~mm}, 68 \mathrm{~mm}$, and $127 \mathrm{~mm}$ for the TEM horn, flat dipole, half-wave dipole, and DRG horn, respectively.

The far-field approximation by curve fitting for the TEM horn and halfwave dipole is plotted in Figs. 2(b)-(d). In the far-field region, the field strength decreases in inverse proportion to the distance $r$ from the antenna. Therefore, as the second condition for the far-field region, the field strength along the distance from the antenna should fit the curve using $1 / r$. In particular, the length of the TEM horn cannot be ignored with respect to the measurement distance; thus, curve fitting was performed using $1 /\left(r+d_{p c}\right)$ considering the phase center. The positions $\left(d_{p c}\right)$ of the phase centers were $76 \mathrm{~mm}, 348 \mathrm{~mm}$, and $313 \mathrm{~mm}$ inside from the aperture at $930 \mathrm{MHz}, 2.45 \mathrm{GHz}$, and $5.8 \mathrm{GHz}$, respectively, as shown in Fig. 1(c). The minimum far-field distance was determined by curve fitting, and then the wave impedance was compared with that of $377 \Omega$ at that distance. The wave impedance of the TEM horn and half-wave dipole at a distance that can be regarded as the far field obtained by curve fitting is close to $377 \Omega$, as shown in Fig. 1(d) and Figs. 2(b)-(d). For example, the minimum far-field distances of the TEM horn are $350 \mathrm{~mm}$ at $930 \mathrm{MHz}, 200 \mathrm{~mm}$ at $2.45 \mathrm{GHz}$, and $50 \mathrm{~mm}$ at $5.8 \mathrm{GHz}$, and the wave impedances at these distances are $368 \Omega, 386 \Omega$, and $368 \Omega$, respectively. The fields at the location where the EUT is placed in the proximity test behave similarly to the near- or far-field region depending on the test frequency and the location when evaluating the field characteristics near the antenna under two far-field conditions on the basis of the propagation characteristics and the wave impedance. However, the far-field distances estimated on the axis of the antenna are different from those under the wellknown far-field condition, which is based on the plane wave, of $2 D^{2} / \lambda$ (where $D$ is the aperture size and $\lambda$ is the wavelength) for aperture antennas, as the fields around antenna are spherical near the antenna.

Figure 3 shows the measured results of the field uniformity of the TEM horn and flat broadband dipole. The TEM horn has a small reduction for the given field strength even near the antenna, for example, the field strength at the distance of $400 \mathrm{~mm}$ at $2.45 \mathrm{GHz}$ is more than $10 \mathrm{~dB}$ higher than that of the flat broadband dipole. Although the flat broadband dipole has two beams spread at $5.8 \mathrm{GHz}$, which results in lower strengths on the axis of the antenna as shown in Fig. 2(d), the TEM horn generated large homogeneous field areas over a wideband compared with the other antennas. Even near the antenna, the field strength of the TEM horn did not change as rapidly as those of dipole-like antennas. 


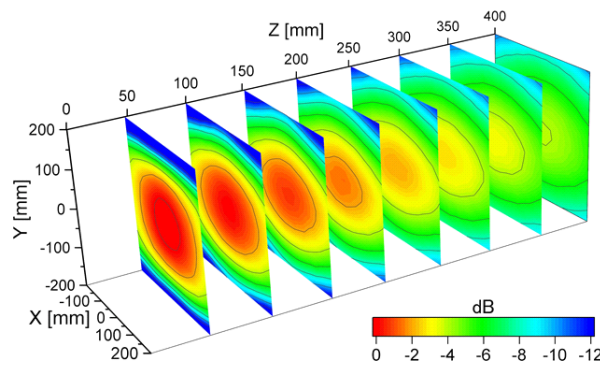

TEM horn

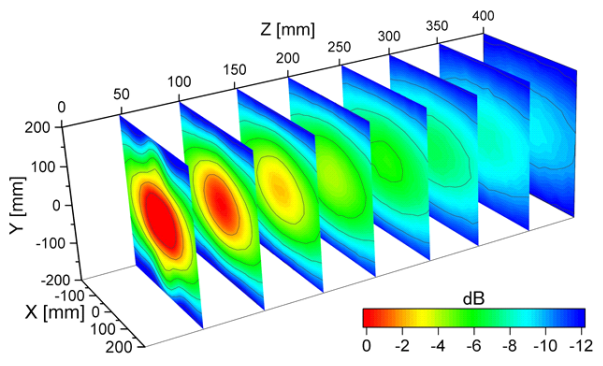

Flat broadband dipole

(a)

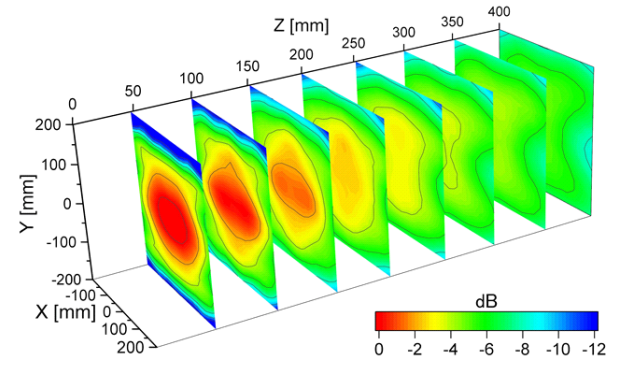

TEM horn

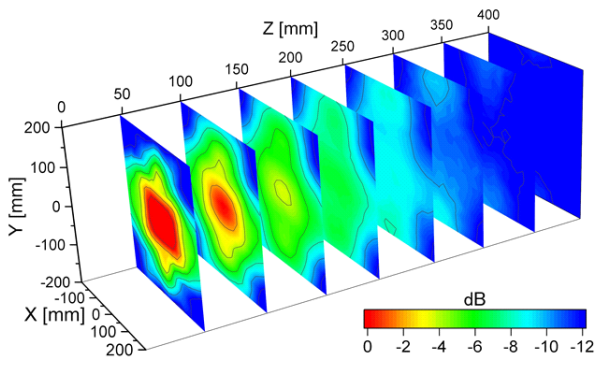

Flat broadband dipole

(b)

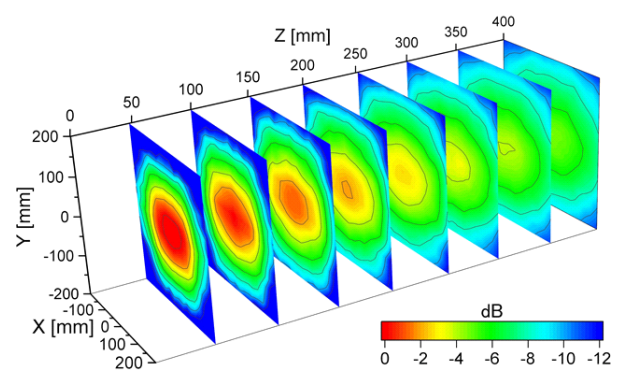

TEM horn

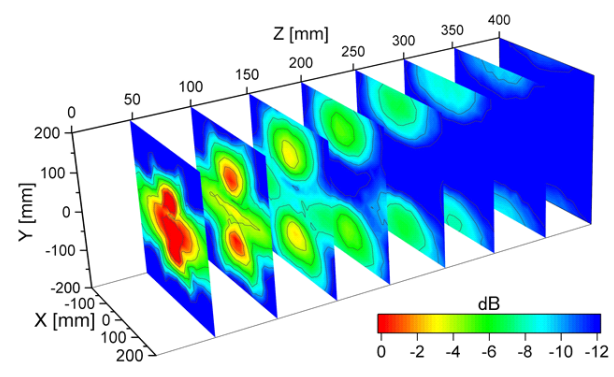

Flat broadband dipole

(c)

Fig. 3: Field distributions of TEM horn and flat broadband dipole at (a) $930 \mathrm{MHz}$, (b) $2.45 \mathrm{GHz}$, and (c) $5.8 \mathrm{GHz}$.

\section{Conclusion}

In near-field immunity tests, TEM horns and various dipole-like antennas are used; however, antenna characteristics affect the test results. The field characteristics near the antenna were compared between the TEM horn that we fabricated and other typical test antennas, namely, a flat broadband dipole, a half-wave dipole, and a DRG horn. The results showed that the propagation characteristics greatly differ among the antennas. In particular, the TEM horn generated a homogeneous field and maintained the field strength with small reduction, whereas the dipole-like antennas showed rapid changes in field strength. In addition, the far-field approximation was performed considering the phase center using the propagation characteristics and the wave impedance, and the behavior of near- or far-field characteristics in proximity of antenna was clarified. 